\title{
Demographic and Socioeconomic Disparities Among Cancer Survivors in Clinical Trials Participation, USA, 2016-2018
}

\author{
Tarang Parekh $^{1}$ (D) Aakash Desai $^{2}$ \\ Published online: 11 June 2020 \\ (C) American Association for Cancer Education 2020
}

\begin{abstract}
The established findings of lower representation of minority population in clinical trials are skewed by the national representation of the US population by race where they account only for 30\%, making it difficult to compare the participation rate by race. We report the direct age-adjusted demographic and socioeconomic disparities in clinical trial participation among cancer survivors using the most recent national survey data. In 2018, 7.1\% of cancer survivors reported participating in the clinical trial as a part of their cancer treatment. The participation rate was significantly higher among younger adults (age 18-24, 19.1\%), male (8.5\%), black (19.8\%), and Hispanic (14.4\%) cancer survivors, while rate was lower among female (4.2\%), and white (5.4\%). By marital status, $8.5 \%$ of unmarried and $3.5 \%$ of married cancer survivors reported clinical trial participation. These results help explain the demographic disparities in cancer survivors and drawing attention to targeted education and awareness intervention for ideal cancer care.
\end{abstract}

Keywords Clinical trial participation $\cdot$ Cancer survivors $\cdot$ Racial disparity $\cdot$ Demographic $\cdot$ Education level

\section{Introduction}

Demographic disparities have been described previously among ethnic minorities who are consistently underrepresented. Previous studies have focused on the representation of racial profile in clinical trials consisting of the overwhelmingly white population [1]. It is not surprising that the White population presentation is higher in clinical trials looking at the US population distribution by race, which reports $76.5 \%$ white population [2]. While we already know about the racial representation, the age-adjusted demographic distribution of cancer survivors participated in clinical trials has not been previously described. The study reports the clinical trial participation rate in cancer survivors among each demographic

Tarang Parekh

tparekh2@gmu.edu; drtarangparekh@gmail.com

1 Department of Health Administration and Policy, George Mason University, Fairfax, VA 22030, USA

2 Department of Medicine, University of Connecticut, Farmington, CT, USA classification, which include age, gender, race, marital status, education, income, and number of cancers.

\section{Method}

Data were analyzed in 2020 from 14 states (Delaware, Georgia, Idaho, Indiana, Kansas, Louisiana, Maryland, Michigan, Missouri, Nebraska, New Jersey, South Dakota, Wisconsin, Virgin Islands) using the optional module on Cancer Survivorship between 2016 and 2018 Behavioral Risk Factor Surveillance System (BRFSS). BRFSS is a population-based annual health survey conducted by the Centers for Disease Control and Prevention (CDC). Individuals with a history of cancer (cancer survivors) were identified if they answered "yes" to any of following questions: "(Ever told) you had skin cancer?" and "(Ever told) you had any other types of cancer?" The sample was restricted to cancer survivors who responded to the question, "Did you participate in a clinical trial as part of your cancer treatment?" $(N=18,084)$. The response was dichotomized and used to calculate the direct age-adjusted prevalence applying the recommended weights to account for the BRFSS complex sampling design [3]. Direct age adjustment was based on the standard 2010 US Census population using age 
categories reported in the figure. Reported observations are unweighted while percentages are weighted. Data were analyzed using STATA-v16.1 (StataCorp).

\section{Result}

Figure 1 displays the clinical trial participation prevalence by cancer survivors' demographics and socioeconomic characteristics. The overall prevalence of clinical trial participation among cancer survivors was $6.8 \%$ with a varied demographic distribution. The prevalence was highest among younger adults aged $18-24$ years $(19.1 \%)$ and among the middleaged group, 45-54 years $(7.8 \%)$. Cancer trial participation was more likely among male $(8.5 \%)$ and less likely among female $(4.2 \%)$. When stratified by race, the prevalence of cancer trial participation was highest among non-Hispanic blacks (19.8\%), lowest among non-Hispanic other including American India/Alaska Native, Hawaiian/Pacific Islander, and Asian (2.8\%). By socioeconomic status, the clinical trial participation rate was lowest among survivors with a college education (4.2-4.3\%) and higher among higher annual income groups $(10.7 \%$ in more than $\$ 50,000$ income group vs. 7.25 in less than $\$ 15,000$ income group). Overall, cancer survivors were more likely to participate if they had three or more concomitant cancers $(20.3 \%)$.

\section{Discussion}

Using the national population-based data, our study found that younger, non-Hispanic black, male cancer survivors with higher annual income $>\$ 50,000$ were more likely to have participated in a clinical trial. Previously, disproportional race representation has been described in clinical trials supporting the US Food and Drug Administration (FDA) oncology drug approvals [1]. Almost three-quarters of the clinical trial participants for such trials were whites while blacks comprised of merely $3.1 \%$, which could be attributed in part to the disproportionate population distribution and cancer incidence in the USA. Our findings could be explained by higher trust in PCPs providing cancer care, monetary compensation, interest in the alternative medicine, or increased awareness about clinical trials in addition to a willingness to participate among the
Fig. 1 Prevalence of cancer clinical trial participation by demographics of cancer survivors, United States, 20162018. D/W/S: Divorced/ Widowed/ Separated. Numbers are unweighted and percentages are weighted

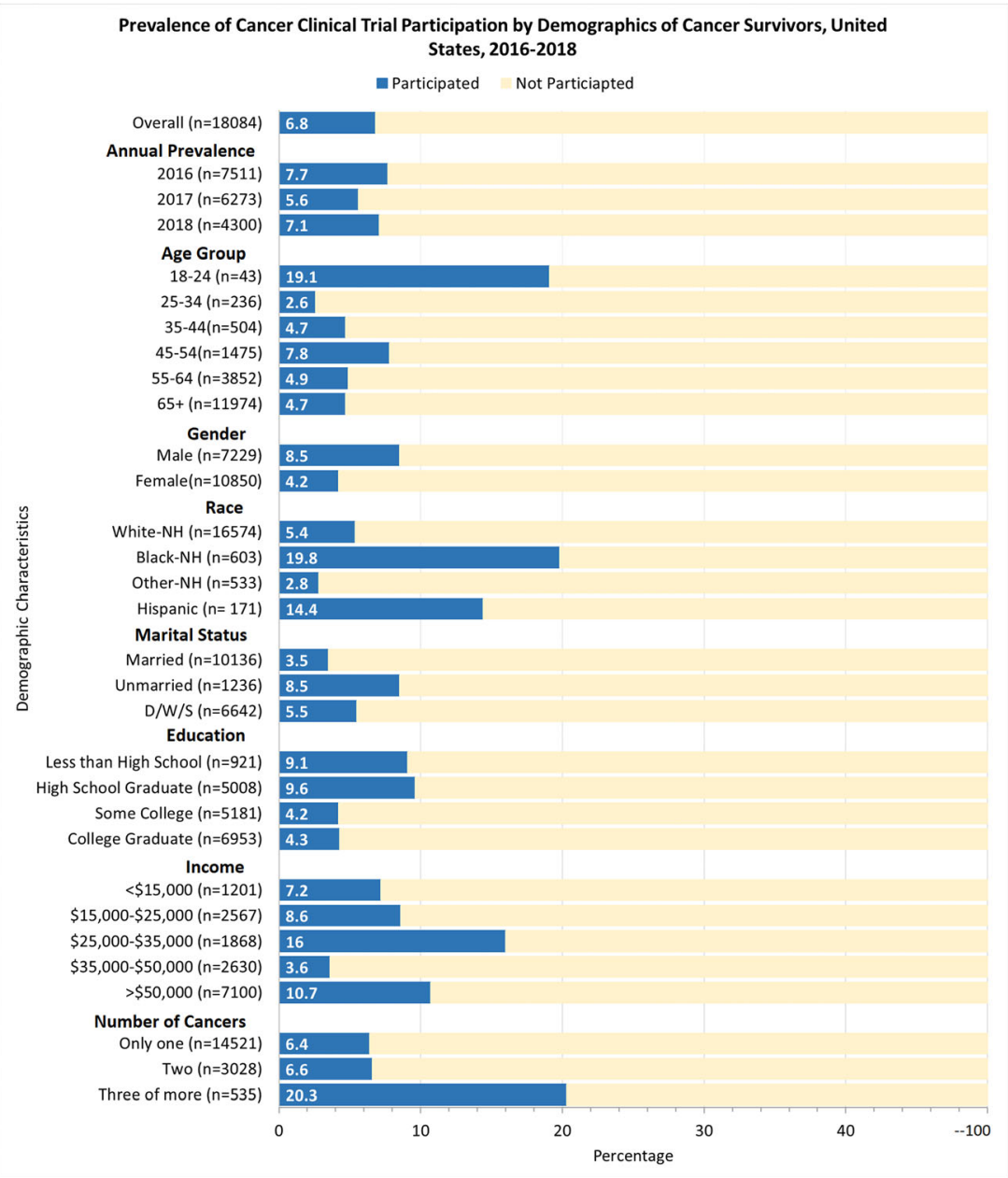


racial minorities [4]. Furthermore, we also found that women, older adults, and cancer survivors of the lower-income group were less likely to have enrolled in clinical trials compared to men, and younger patients, and high-income group respectively, which is consistent with studies of clinical trial enrollments $[5,6]$.

In our analysis, we found that survivors with high school or lesser education were more likely to participate compared to those with college education. A study using internet-based treatment decision tool found that patients with lower education were less likely to participate in a cancer clinical trial [6]. However, this study was limited to breast, colorectal, lung, or prostate cancer treatment and suffered from the small sample size. In contrast, our study had considered a broader definition of cancer survivors with a larger sample size representative of the national average. Although, counter-intuitively, a lower participation rate could also reflect the skeptical sentiment regarding clinical trials among the well-educated. Clinical trial enrollment for non-cancer studies has previously described no influence of education [7]. However, our study is the first reporting on extensive data on disparities in clinical trial enrollment for cancer survivors. Given these findings, we believe that future studies will need to further elucidate the influence of education on clinical trial participation in cancer clinical trials, which may have important implications in targeting enrollment and policymaking.

Educational awareness regarding cancer clinical trial participation is even more critical with current COVID-19 pandemic, which has shown a serious disruptive impact on conducting cancer clinical trials with probable long-term consequences [8]. Education level could potentially play an important role as the level of understanding for the magnitude of the situation may differ that could widen those existing disparities in cancer clinical trial participation.

Limitations include lack of information on the phase of clinical trial participation, completion or dropout rate, the reason for participating in clinical trials, and possible recall bias in the survey data. Despite this, our study provided information at a national level using population-based data. The large sample size of the cancer survivor population cohort enabled us to study accurate estimates.

\section{Conclusion}

Despite several efforts, health care disparities persist in oncology trials. Women and the elderly continue to be underrepresented when compared to the US population. Whether the rationale for including racial minorities in cancer clinical trials is framed in terms of skewed representations, our study findings suggest that equitable participation is possible among different gender, race, education level, and economic classes with targeted education and awareness intervention as health care equity is a necessity to fulfill an ethical and scientific ideal in cancer care.

Author Contributions All the authors contributed equally and have reviewed and approved the final manuscript as submitted.

\section{Compliance with Ethical Standards}

Conflict of Interest The authors declare that they have no conflict of interest.

\section{References}

1. Loree JM, Anand S, Dasari A, Unger JM, Gothwal A, Ellis LM, Varadhachary G, Kopetz S, Overman MJ, Raghav K (2019) Disparity of race reporting and representation in clinical trials leading to cancer drug approvals from 2008 to 2018. JAMA Oncol 5: e191870-e191870. https://doi.org/10.1001/jamaoncol.2019.1870

2. U.S. Census Bureau QuickFacts: United States. https://www.census. gov/quickfacts/fact/table/US/IPE120218. Accessed 22 Mar 2020

3. Centers for Disease Control and Prevention (2019) Behavioral risk factor surveillance system: complex sampling weights and preparing 2018 BRFSS Module Data for Analysis. Accessed at https://www. cdc.gov/brfss/annual_data/2018/pdf/Complex-Smple-WeightsPrep-Module-Data-Analysis-2018-508.pdf on February, 2020

4. Langford A, Resnicow K, An L (2010) Clinical trial awareness among racial/ethnic minorities in HINTS 2007: sociodemographic, attitudinal, and knowledge correlates. J Health Commun 15:92-101. https://doi.org/10.1080/10810730.2010.525296

5. Murthy VH, Krumholz HM, Gross CP (2004) Participation in cancer clinical trials: race-, sex-, and age-based disparities. JAMA 291: 2720-2726. https://doi.org/10.1001/jama.291.22.2720

6. Unger JM, Gralow JR, Albain KS, Ramsey SD, Hershman DL (2016) Patient income level and cancer clinical trial participation: a prospective survey study. JAMA Oncol 2:137-139. https://doi.org/ 10.1001/jamaoncol.2015.3924

7. Corbie-Smith G, Viscoli CM, Kernan WN, Brass LM, Sarrel P, Horwitz RI (2003) Influence of race, clinical, and other sociodemographic features on trial participation. J Clin Epidemiol 56: 304-309. https://doi.org/10.1016/S0895-4356(03)00046-5

8. Saini KS, de las Heras B, de Castro J et al (2020) Effect of the COVID-19 pandemic on cancer treatment and research. Lancet Haematol 7:e432-e435. https://doi.org/10.1016/S2352-3026(20) 30123-X

Publisher's Note Springer Nature remains neutral with regard to jurisdictional claims in published maps and institutional affiliations. 